\title{
Sedentary Lifestyle and Cardiovascular Health
}

\author{
Soo Young Kim* \\ Department of Family Medicine, Kangdong Sacred Heart Hospital, Hallym University College of Medicine, Seoul, Korea
}

A sedentary lifestyle can be defined as "a type of lifestyle with little or no physical activity".1) Sedentary behavior includes reading, computer use, watching television, office work, and cell phone use. A similar but somewhat different term is screen time. This is the sum of the time spent viewing a television, computer monitor, mobile device, or other screen. ${ }^{2)}$ Prospective evidence is accumulating that sedentary behavior can be a risk factor for the morbidity and mortality of cardiovascular disease and diabetes mellitus, and for all-cause mortality.

In the present issue, Park et al. ${ }^{4)}$ evaluated the association between sedentary time and cardiovascular risk factors in Korean adults using the 2013 Korea National Health and Nutrition Examination Survey data. The authors found that after adjustment for body mass index, waist circumference, moderate-to-vigorous physical activity, and other variables, prolonged sedentary time was significantly associated with high diastolic blood pressure and low high-density lipoprotein cholesterol level.

The authors also reported that the mean sedentary time among participants was 6.1 hours per day, or 42.7 hours per week. It was reported that the average sedentary time increased from 26 hours per week in 1965 to 38 hours per week in 2009 in the United States and from 30 hours per week in 1960 to 42 hours per week in 2005 in the United Kingdom. ${ }^{5)}$ In light of these data, the sedentary time in Korea seems to be higher than that in other countries, and may adversely affect future health.

However, there is no "gold standard" for sedentary behavior assessment. Self-reporting measures provide information on the behavioral context that is not available from objective measures. The evidence to date is suggestive, but not conclusive, that sedentary behavior contributes to cardiovascular disease and diabetes mellitus risk. Therefore, more research and data are needed to develop public health interventions and recommendations.

\section{CONFLICT OF INTEREST}

No potential conflict of interest relevant to this article was reported.

\section{REFERENCES}

1. Gonzalez K, Fuentes J, Marquez JL. Physical inactivity, sedentary behavior and chronic diseases. Korean J Fam Med 2017; 38:111-5.

2. Laurson KR, Eisenmann JC, Welk GJ, Wickel EE, Gentile DA, Walsh DA. Combined influence of physical activity and screen time recommendations on childhood overweight. J Pediatr 2008;153:209-14.

3. Endorsed by The Obesity Society, Young DR, Hivert MF, Alhassan S, Camhi SM, Ferguson JF, et al. Sedentary behavior and cardiovascular morbidity and mortality: a science advisory from the American Heart Association. Circulation 2016;134:e262-79.

4. Park JH, Joh HK, Lee GS, Je SJ, Cho SH, Kim SJ, et al. Association between sedentary time and cardiovascular risk factors in Korean adults. Korean J Fam Med 2018;39:29-36.

5. Ng SW, Popkin BM. Time use and physical activity: a shift away from movement across the globe. Obes Rev 2012;13: 659-80. 